\title{
BMJ Open Tolerability of statin-based management of patients with a history of statin- associated muscle symptoms: protocol for a systematic review
}

\author{
Fanny Villoz (D , ${ }^{1,2}$ Christina Lyko, ${ }^{1}$ Cinzia Del Giovane, ${ }^{1}$ Nicolas Rodondi, ${ }^{1,2}$ \\ Manuel R Blum ${ }^{1,2}$
}

To cite: Villoz F, Lyko C, Del Giovane C, et al. Tolerability of statin-based management of patients with a history of statin-associated muscle symptoms: protocol for a systematic review. BMJ Open 2021;11:e052341. doi:10.1136/ bmjopen-2021-052341

- Prepublication history and additional supplemental material for this paper are available online. To view these files, please visit the journal online (http://dx.doi.org/10.1136/ bmjopen-2021-052341).

Received 13 April 2021 Accepted 02 July 2021

Check for updates

(C) Author(s) (or their employer(s)) 2021. Re-use permitted under CC BY-NC. No commercial re-use. See rights and permissions. Published by BMJ.

${ }^{1}$ Institute of Primary Health Care (BIHAM), University of Bern,

Bern, Switzerland

${ }^{2}$ Department of General Internal

Medicine, Inselspital, Bern

University Hospital, University of Bern, Bern, Switzerland

Correspondence to

Dr Manuel R Blum;

manuel.blum@biham.unibe.ch

\section{ABSTRACT}

Introduction Statin-associated muscle symptoms

(SAMSs) are a major clinical issue in the primary and secondary prevention of cardiovascular events. Current guidelines advise various approaches mainly based on expert opinion. We will lead a systematic review and meta-analysis to explore the tolerability and acceptability and effectiveness of statin-based therapy management of patients with a history of SAMS. We aim to provide evidence on the tolerability and different strategies of statin-based management of patients with a history of SAMS.

Methods and analysis We will conduct a systematic review of randomised controlled trials (RCTs) and nonrandomised studies with a control group. We will search in Data sources MEDLINE, EMBASE, Cochrane Central Register of Controlled Clinical Trials, Scopus, Clinicaltrials. gov and Proquest from inception until April 2021. Two independent reviewers will carry out the study selection based on eligibility criteria. We will extract data following a standard data collection form. The reviewers will use the Cochrane Collaboration's tools and Newcastle-0ttawa Scale to appraise the study risk of bias. Our primary outcome will be tolerability and our secondary outcomes will be acceptability and effectiveness. We will conduct a qualitative analysis of all included studies. In addition, if sufficient and homogeneous data are available, we will conduct quantitative analysis. We will synthesise dichotomous data using OR with $95 \% \mathrm{Cl}$ and continuous outcomes by using mean difference or standardised mean difference (with $95 \% \mathrm{Cl}$ ). We will determine heterogeneity visually with forest plots and quantitatively with $\mathrm{I}^{2}$ and $\mathrm{Q}$ test. We will summarise the confidence in the quantitative estimate by using Grading of Recommendations Assessment, Development and Evaluation approach. Ethics and dissemination As a systematic review of literature without collection of new clinical data, there will be no requirement for ethical approval. We will disseminate findings through peer-reviewed publications. PROSPERO registration number CRD42020202619.

\section{INTRODUCTION}

Statin-associated muscle symptoms (SAMSs), a composite of muscle symptoms appearing consequent to the initiation or the increase
Strengths and limitations of this study

- This systematic review protocol observes the Preferred Reporting Items for Systematic Review and Meta-Analyses Protocols checklist.

- This systematic review will be the first to systematically review tolerability of statin-based management in patients with a history of statin-associated muscle symptom.

- This systematic review will provide the highest level of evidence for clinical decisiveness due to the inclusion of randomised controlled trials (RCTs) as well as non-RCTs.

Heterogeneity of the studies may not permit a quantitative analysis.

of a statin's treatment, ${ }^{1}$ are a major clinical issue in the primary and secondary prevention of cardiovascular events. Statins are a cornerstone in the prevention of cardiovascular risk and mortality, ${ }^{2}{ }^{3}$ and are widely prescribed with increased intensity to achieve currently recommended low-density lipoprotein cholesterol (LDL-C) levels. ${ }^{4-6}$ Nevertheless, SAMS, a commonly reported muscle symptom, threatens the ability of a significant proportion of patients to tolerate evidencebased dosing: based on observational data or registries reported by patients. SAMS affects between $5 \%$ and $29 \%$ of statin-treated individuals. ${ }^{1}$ This lack of tolerability is associated with higher cardiovascular disease risk. ${ }^{6}$ Meanwhile, the high cost of the non-statin alternative drugs, such as PCSK9 inhibitors, and the lack of other effective alternatives, remains a concern, ${ }^{78}$ and statins currently remain the main treatment option.

The European Atherosclerosis Society Consensus Panel Statement recommend multiple different strategies to manage patients with SAMS but they are based only on experts' opinion due to lack of sufficient data. ${ }^{1}$ 
A systematic review and meta-analysis of 12 randomised controlled trials (RCTs) and one quasi-RCT on the efficacy and safety of alternate day versus daily dosing of statins with participants without previous SAMS in 2017 found a statistically non-significant difference in terms of change in LDL-C in both groups and concluded good adherence and tolerability of both treatment. ${ }^{9}$ However, this meta-analysis has not assessed the specific population of patients suffering from SAMS. A systematic review of three case reports, five retrospective studies, one prospective study and one randomised trial assessing effectiveness of intermittent non-daily administration of statin strategies with patients with previous statin-induced myopathy in 2013 found that $70 \%$ of patients could tolerate an intermittent dosing strategy and concluded that uncertainty remains and that larger scale randomised trials are required. ${ }^{10}$

Since publication of these systematic reviews, new evidence on the management of SAMS has emerged both from RCTs and observational data. ${ }^{11-16}$ Therefore, we decided to conduct a systematic review and meta-analysis to investigate the tolerability, acceptability and effectiveness of statin-based therapy management in patients with a history of SAMS compared with all available comparators. In a patient-centred perspective, we will focus on the tolerability as the primary outcome. We will not only include intermittent dosing strategies, but also other strategies to broaden our conclusion. Our systematic review and meta-analysis will be complementary to the ongoing meta-analysis ${ }^{17}$ on statin adverse events with the particularity to focus on patients with a history of SAMS and SAMS' management.

We aim to provide quality evidence for the tolerability of statin-based management of patients with a history of SAMS. We will also highlight gaps in available evidence to direct further research.

\section{METHODS AND ANALYSIS \\ Eligibility criteria \\ Types of studies}

We will include human RCTs and prospective and retrospective cohort studies with a control group, published in English from inception until April 2021. There will be no follow-up length or setting restriction. We will include relevant studies mainly based on the population and intervention criteria to avoid exclusion of studies which poorly report tolerability or adverse outcomes in their titles and abstracts. In the case of multiple publications from the same study, we will include the report with the most relevant data relating to our interest.

We will differentiate between the absence of muscle symptoms and the absence of reporting muscle symptoms and include data only from studies reporting the absence of muscle symptoms. We will include post-hoc analysis of prior RCT so long as there is a comparison group.

\section{Types of participants}

We will include studies examining adult humans previously on statins with a history of SAMS. We will also include studies examining adults previously on statins with a history of statin intolerance without precision of SAMS or other intolerance. Indeed, SAMS is a type of statin intolerance concerning specifically muscle symptoms. Nevertheless, some participants can also report other types of intolerance, as for example, impaired cognition, ${ }^{18}$ hepatic dysfunction ${ }^{19}$ or depression. ${ }^{20}$ SAMS is also a recent definition and could have been reported as 'intolerance' or 'muscle-related adverse events' in the past.

We will exclude studies examining adults without a history of statin intolerance, children, adolescents and pregnant women.

\section{Types of interventions}

We defined statin-based therapy management of patients with a history of SAMS as all statin management strategies with the aim of optimal lipid profile lowering and decreasing adverse effects. Examples include statin continuation, re-challenge, up-titration, down-titration, second statin at the usual or starting dose, low dosing of a high-intensity statin, intermittent dosing statin. All the variations of statin management strategies will be included, for example the variation in dosage, intensity, frequency of delivery, duration of delivery and timing of delivery. All co-lifestyle modifications (exercise and diet) and all additional interventions in the intervention group are included if present in the control group too.

\section{Types of comparators}

Placebo, usual care, other statins regimens, other nonstatin lipid-lowering drug regimens (ezetimibe, PCSK9 inhibitors), statin regimens with additional interventions (statins with ezetimibe, CoQ10, vitamin D) or no treatment.

\section{Types of outcomes}

Tolerability, as defined in individual studies, will be our main outcome. We anticipate that the proportion of population with muscle symptoms-related adverse events compared with control group would be the most feasible measure to analyse. If reported, we will collect time until muscle symptoms-related adverse events and measure the adverse event rate.

We defined adverse event as 'an unfavorable outcome that occurs during or after the use of a drug or other intervention and the causal relation between the intervention and the event is at least a reasonable possibility' as defined in the Cochrane handbook for systematic reviews of interventions. $^{21}$

Acceptability, as defined in individual studies, will be a secondary outcome. We anticipate that the proportion of population with muscle symptoms-related study or treatment discontinuation compared with control group will be the most feasible measure to analyse. 
Effectiveness, defined as the change in lipid profile will be a secondary outcome. The lipid profile includes at least LDL-C in addition to total cholesterol, high-density lipoprotein cholesterol (HDL-C) and triglycerides as provided by the individual studies.

\section{Search strategy and study selection \\ Search strategy}

The first author will develop a search strategy for each database included MEDLINE, EMBASE and Cochrane Central Register of Controlled Clinical Trials in cooperation with a trained librarian using computerised search (see online supplemental file 1). Search terms and syntax will be adapted for each specific database. Moreover, we will complete a hand search using forward and backwards citations, and evaluate the grey literature (Scopus, Clinicaltrials.gov and Proquest) for additional potentially relevant and unpublished articles. We will search only studies written in English.

\section{Study selection}

Two trained reviewers (FV and CL) will evaluate independent eligibility based on titles and abstracts of all studies retrieved in our electronic search. ${ }^{21}$ We will upload the literature search results to Rayyan QCRI, an internetbased software programme that facilitates collaboration among reviewers during the selection process. We will remove duplicates using reference management software. The two reviewers will assess the remaining studies for inclusion after full-text evaluation. If studies do not report muscle symptoms events or discontinuation in the full text, we will request them from the authors. We will then include or exclude the study, depending on the information provided. We will still include studies with absence of reporting muscles symptoms if they present data of interest for secondary outcomes. We will include post-hoc analysis of prior RCT so long as there is a comparison group.

We will list excluded full-text studies together with the reason for exclusion. We will resolve discrepancies by making a consensus among the study team. If we cannot reach consensus, we will consult a third reviewer. We will follow the Preferred Reporting Items for Systematic Review and Meta-Analyses recommendations to summarise the study selection. We will document all decisions made in the study selection process.

\section{Data extraction and management}

We will manage data with an online shared data form among the review team.

We will use a standard data collection form, piloted by the review team using representative sample of included studies. Two reviewers will manage data in duplicate. We will request additional data from the authors by email. The two reviewers will discuss and resolve disagreements by consensus or consult a third reviewer.

The data collection items are listed in table 1.

\section{Quality assessment}

Two reviewers will autonomously evaluate the risk of bias of each study. We will use the Cochrane Collaboration tools for appraising the risk of bias of prospective studies and the Newcastle-Ottawa scale for the retrospective

\begin{tabular}{|c|c|}
\hline Method & $\begin{array}{l}\text { Study design. } \\
\text { Participants. } \\
\text { Sample size, loss to follow-up. } \\
\text { Characteristics of participants at baseline as age, sex, body mass index, cardiovascular comorbidities, } \\
\text { cardiovascular risk factors, co-medications with influence on the cytochrome of interest, history of adverse } \\
\text { reaction to multiple medications, lipid profile, past achievement of LDL-C goals; creatine kinase, liver function } \\
\text { test, intolerance, SAMS. }\end{array}$ \\
\hline $\begin{array}{l}\text { Intervention and } \\
\text { control }\end{array}$ & $\begin{array}{l}\text { Statins, doses, timing, frequency, length of intervention, washout period, duration of follow-up. } \\
\text { Description of co-interventions, lifestyle modification, modification of baseline medication regimen. } \\
\text { Types of comparator, doses, timing, frequency, intervention protocols, length of intervention, washout period, } \\
\text { duration of follow-up. }\end{array}$ \\
\hline Outcomes & $\begin{array}{l}\text { Proportion of population with/without muscles symptoms-related adverse events, time to muscles symptoms- } \\
\text { related adverse events, proportion of population with muscles symptoms related drop out, lipid profile, creatine } \\
\text { kinase level, liver function test. } \\
\text { Multiple adverse events occurrence in the same individuals. } \\
\text { All other adverse outcomes and collection systematic: definition of each adverse outcome addressed, method } \\
\text { of ascertainment (patient report vs active search), method of measurement, timing and frequency of adverse } \\
\text { events, measurement of the severity. } \\
\text { Associated factor to the adverse events. } \\
\text { For each outcome at each time point: number of participants randomly assigned and included in the analysis; } \\
\text { number of participants who withdrew, were lost to follow-up or were excluded with reasons for each. }\end{array}$ \\
\hline Notes & Conflicts of interest, funding sources. \\
\hline
\end{tabular}

SAMS, statin-associated muscle symptom. 
studies. Discrepancy among the two reviewers will be solved by consensus or by a third person.

On the meta-bias level, a sensitivity analysis to determine the effect of selective reporting will also be considered. If data permits, we will assess small study effect via funnel plots and formally with the Egger test.

\section{Data synthesis and statistical analyses}

We will synthesise the systematic review qualitatively and quantitatively.

In the qualitative synthesis, we will summarise the characteristics and findings of the included studies in text and tables. We will categorise our summaries of studies according to type of intervention, comparator, outcome and study design. We will present the limitations of the included studies and recommendations for future research.

In the quantitative synthesis, we will perform study level meta-analysis if studies are sufficiently homogenous and if enough data are available.

We will use the same summary measures in RCT and non-randomised trials. For tolerability and acceptability, we will synthesise dichotomous data using OR with $95 \%$ CI. For efficacy, we will synthesise the change in lipid profile from baseline as a continuous outcome using mean difference or standardised mean difference (with 95\% CI) depending on the different metrics.

When studies reported the number of muscle events instead of the number of subjects experiencing muscular event, we will contact the authors to request the number of patients with $>0$ events. If this could not be addressed, we will make the assumption of one event per subject. If a group of the studies reported zeroes event, we will use the zero count-cell method to allow statistical measures. We will exclude studies with zero events in both groups from the analysis.

Concerning acceptability, if the measure to analyse is the proportion of population with muscle symptoms-related discontinuation compared with the control group, we will assess if the participant was still blinded to treatment attribution before the discontinuation to avoid bias.

Regarding the variety of study designs, we will first pool data, then analyse data from different type of studies separately (eg, RCT vs non-randomised control studies, crossover trial vs parallel trial). We will collect the variables used for the adjustment in each study. When dealing with crossover trials, data after the crossover will be analysed.

We will assess heterogeneity visually with forest plots, $\mathrm{I}^{2}$ test and the Q-test. We will assess and interpret heterogeneity in line with the guidance in the Cochrane handbook of systematic reviews and meta-analysis. In case of heterogeneity, we will explore potential sources in subgroup analyses. In the case of significant heterogeneity, we will use the random effect model.

We will conduct subgroup analysis to explore possible sources of heterogeneity: pre-planned variables to explore are primary versus secondary prevention, high intensity versus non-high intensity statins, intermittent dosing versus daily dosing, patients with a history of SAMS versus patients with a history of statin intolerance, only statins intervention versus statins and additional interventions and participants with versus without a statin at inclusion. If feasible, we will consider the different follow-up times in a meta-regression.

We will conduct the analysis using STATA V.16 software (StataCorp, College Station, Texas, USA).

We will recapitulate the confidence we have in the resulting body of evidence using Grading of Recommendations Assessment, Development and Evaluation working group methodology.

\section{ETHICS AND DISSEMINATION}

We plan to publish the review in a clinical journal from the relevant field (endocrinology, cardiology and internal medicine).

\section{PATIENT AND PUBLIC INVOLVEMENT}

Patients and/or the public were not involved in the design or conduct or reporting or dissemination plans of this research.

Acknowledgements We thank Miss Tania Rivero, medical information specialist from the University of Bern for her help in developing the search strategy.

Contributors FV, MB and NR designed the topic. FV carried out background exploratory searches. FV elaborated the search strategy. FV wrote the protocol. MB, $\mathrm{CDG}$ and NR gave critical appraisal and senior control. For the systematic review, FV and LC will accomplish the searches, data extraction and risk of bias assessment. FV will execute the analysis. MB will supervise the searches, data analysis and extraction. CDG will supply statistical expertise for data analysis. All authors have approved the publication of the protocol after cautious reading.

Funding The authors have not declared a specific grant for this research from any funding agency in the public, commercial or not-for-profit sectors.

Competing interests None declared.

Patient consent for publication Not required.

Provenance and peer review Not commissioned; externally peer reviewed.

Supplemental material This content has been supplied by the author(s). It has not been vetted by BMJ Publishing Group Limited (BMJ) and may not have been peer-reviewed. Any opinions or recommendations discussed are solely those of the author(s) and are not endorsed by BMJ. BMJ disclaims all liability and responsibility arising from any reliance placed on the content. Where the content includes any translated material, BMJ does not warrant the accuracy and reliability of the translations (including but not limited to local regulations, clinical guidelines, terminology, drug names and drug dosages), and is not responsible for any error and/or omissions arising from translation and adaptation or otherwise.

Open access This is an open access article distributed in accordance with the Creative Commons Attribution Non Commercial (CC BY-NC 4.0) license, which permits others to distribute, remix, adapt, build upon this work non-commercially, and license their derivative works on different terms, provided the original work is properly cited, appropriate credit is given, any changes made indicated, and the use is non-commercial. See: http://creativecommons.org/licenses/by-nc/4.0/.

\section{ORCID iD}

Fanny Villoz http://orcid.org/0000-0002-4626-5106

\section{REFERENCES}

1 Stroes ES, Thompson PD, Corsini A, et al. Statin-associated muscle symptoms: impact on statin therapy-European atherosclerosis 
Society consensus panel statement on assessment, aetiology and management. Eur Heart J 2015;36:1012-22.

2 Bakker-Arkema RG, Davidson MH, Goldstein RJ, et al. Efficacy and safety of a new HMG-CoA reductase inhibitor, atorvastatin, in patients with hypertriglyceridemia. JAMA 1996;275:128-33.

3 Hunninghake DB, Stein EA, Bays HE, et al. Rosuvastatin improves the atherogenic and atheroprotective lipid profiles in patients with hypertriglyceridemia. Coron Artery Dis 2004;15:115-23.

4 The Lancet. Statins for millions more? Lancet 2014;383:669.

5 Grundy SM, Stone NJ, Bailey AL, et al. 2018 AHA/ACC/AACVPR/ AAPA/ABC/ACPM/ADA/AGS/APhA/ASPC/NLA/PCNA guideline on the management of blood cholesterol: a report of the American College of Cardiology/American Heart Association Task Force on clinical practice guidelines. J Am Coll Cardiol 2019;73:e285-350.

6 Piepoli MF, Hoes AW, Agewall S, et al. 2016 European guidelines on cardiovascular disease prevention in clinical practice: the Sixth Joint Task Force of the European Society of Cardiology and other societies on cardiovascular disease prevention in clinical practice (constituted by representatives of 10 societies and by invited experts) Developed with the special contribution of the European Association for Cardiovascular Prevention \& Rehabilitation (EACPR). Eur Heart J 2016;37:2315-81.

7 Cannon CP, Blazing MA, Giugliano RP, et al. Ezetimibe added to statin therapy after acute coronary syndromes. N Engl J Med 2015;372:2387-97.

8 Tice JA, Kazi DS, Pearson SD. Proprotein convertase subtilisin/kexin type 9 (PCSK9) inhibitors for treatment of high cholesterol levels: effectiveness and value. JAMA Intern Med 2016;176:107-8.

9 Awad K, Mikhailidis DP, Toth PP, et al. Efficacy and safety of alternate-day versus daily dosing of statins: a systematic review and meta-analysis. Cardiovasc Drugs Ther 2017;31:419-31.

10 Keating AJ, Campbell KB, Guyton JR. Intermittent nondaily dosing strategies in patients with previous statin-induced myopathy. Ann Pharmacother 2013;47:398-404.
11 Nissen SE, Stroes E, Dent-Acosta RE, et al. Efficacy and tolerability of evolocumab vs ezetimibe in patients with muscle-related statin intolerance: the GAUSS-3 randomized clinical trial. JAMA 2016;315:1580-90

12 Taylor BA, Lorson L, White CM, et al. A randomized trial of coenzyme Q10 in patients with confirmed statin myopathy. Atherosclerosis 2015;238:329-35.

13 Joy TR, Monjed A, Zou GY, et al. N -of-1 (Single-Patient) trials for statin-related Myalgia. Ann Intern Med 2014;160:301-310-10.

14 Kristiansen O, Vethe NT, Peersen K, et al. Effect of atorvastatin on muscle symptoms in coronary heart disease patients with selfperceived statin muscle side-effects: a randomized, double blinded crossover trial. Eur Heart J Cardiovasc Pharmacother 2020;01:01.

15 Wood FA, Howard JP, Finegold JA, et al. N-Of-1 trial of a statin, placebo, or no treatment to assess side effects. N Engl J Med Overseas Ed 2020;383): :2182-4.

16 Herrett E, Williamson E, Brack K, et al. Statin treatment and muscle symptoms: series of randomised, placebo controlled n-of-1 trials. BMJ 2021;372:n135

17 Reith C, Blackwell L, Emberson J, et al. Protocol for analyses of adverse event data from randomized controlled trials of statin therapy. Am Heart J 2016;176:63-9.

18 Evans MA, Golomb BA. Statin-associated adverse cognitive effects: survey results from 171 patients. Pharmacotherapy 2009;29:800-11.

19 Björnsson E, Jacobsen El, Kalaitzakis E. Hepatotoxicity associated with statins: reports of idiosyncratic liver injury post-marketing. $J$ Hepatol 2012;56:374-80.

$20 \mathrm{Kang} \mathrm{J}-\mathrm{H}$, Kao L-T, Lin $\mathrm{H}-\mathrm{C}$, et al. Statin use increases the risk of depressive disorder in stroke patients: a population-based study. $J$ Neurol Sci 2015;348:89-93.

21 Lefebvre C, Glanville J, Briscoe S, et al. Searching for and selecting studies. Cochrane Handbook for systematic reviews of interventions 2019:67-107. 